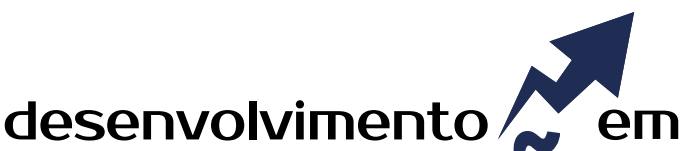 QUESTÃO
}

\section{Efeito do Acesso aos Recursos no Desempenho da Empresa Evidências para o Setor Metalomecânico no Contexto de Aglomeração Territorial}

\author{
http://dx.doi.org/10.21527/2237-6453.2020.53.288-306
}

Recebido em: 29/2/2020

Aceito em: 19/8/2020

\section{Claudionor Guedes Laimer, ${ }^{1}$ Adrieli Cecchin Fortuna, ${ }^{2}$ Viviane Rossato Laimer ${ }^{3}$}

\begin{abstract}
RESUMO
A aglomeração territorial contribui para facilitar o acesso aos recursos, proporcionado à empresa um desempenho superior aos concorrentes, o que pode criar e/ou manter uma vantagem competitiva. Nessa perspectiva, este estudo tem como objetivo investigar o efeito do acesso aos recursos no desempenho das empresas do setor metalomecânico no contexto de aglomeração territorial. Para tanto, realizou-se uma pesquisa quantitativa, do tipo descritiva, sendo realizada uma survey com 73 empresas do setor metalomecânico. $\mathrm{Na}$ análise dos dados utilizou-se a técnica de regressão linear múltipla para evidenciar o efeito do acesso aos recursos no desempenho. Os resultados indicam que os recursos físicos e organizacionais influenciam positivamente no desempenho das empresas, ao passo que os recursos financeiros e humanos não apresentaram influência significativa no desempenho em um contexto de aglomeração territorial. O estudo contribui com a literatura, evidenciando que a facilidade de acesso aos recursos pode proporcionar um desempenho superior e, também, indicando que os recursos apresentam diferentes tipos de relação com o desempenho. Desta forma, a pesquisa tem implicações gerenciais - possibilita que os gestores identifiquem os recursos que podem ser fonte de vantagem competitiva - e governamentais - proporciona subsídios aos governantes na elaboração de políticas públicas, priorizando estímulos ou incentivos aos tipos de recursos que possam proporcionar um aumento na competitividade das empresas.
\end{abstract}

Palavras-chave: Recursos. Acesso aos recursos. Desempenho. Aglomeração territorial. Setor metalomecânico.

ACCESS TO RESOURCES EFFECT ON FIRM PERFORMANCE:

EVIDENCE FOR THE METAL-MECHANICAL SECTOR IN THE CONTEXT OF TERRITORIAL AGGLOMERATION

\begin{abstract}
Territorial agglomeration contributes to facilitate access to resources, providing the company with a performance superior to that of its competitors, which can create and / or maintain a competitive advantage. Thus, this study aims to investigate the effect of access to resources on the firm performance in the metal-mechanical sector, in the context of territorial agglomeration. For that, a quantitative research, of the descriptive type, was carried out, being carried through a survey with 73 firm of the metal-mechanic sector. In the data analysis, the multiple linear regression technique was used to show the effect of access to resources on performance. The results indicate that physical and organizational resources positively influence the performance of companies, while financial and human resources did not have a significant influence on performance in a context of territorial agglomeration. The study contributes to the literature, showing that ease of access to resources can provide superior performance and also indicating that resources have different types of relationship with performance. In this way, the study has managerial implications - it allows managers to identify resources that can be a source of competitive advantage - and governmental ones - it provides subsidies to governments in the elaboration of public policies, prioritizing stimuli or incentives to the types of resources that can enable an increase competitiveness of companies.
\end{abstract}

Keywords: Resources. Access to resources. Performance. Territorial agglomeration. Metal-mechanic sector.

\footnotetext{
${ }^{1}$ Doutor em Administração pela Universidade do Vale do Rio dos Sinos (Unisinos). Professor da Faculdade Meridional (Imed). http://lattes. cnpq.br/3904741903410593. https://orcid.org/0000-0001-5696-8043. claudionorlaimer@gmail.com

${ }^{2}$ Mestre em Administração pela Faculdade Meridional (Imed). http://lattes.cnpq.br/1926550636384402. https://orcid.org/0000-0003-06655502. adrielicecchin@hotmail.com

${ }^{3}$ Doutora em Desenvolvimento Regional pela Universidade de Santa Cruz do Sul (Unisc). Professora da Universidade Federal da Fronteira Sul (UFFS). http://lattes.cnpq.br/0443414851457110. https://orcid.org/0000-0002-5828-8209. vivianelaimer@gmail.com
} 
Em um ambiente dinâmico e competitivo, com inúmeros concorrentes concentrados e dispersos geograficamente, o desempenho tem sido fator determinante para a sobrevivência das empresas no mercado. Assim sendo, a aglomeração territorial passou a ser fundamental, porque pode possibilitar uma melhoria no desempenho da empresa em relação aos concorrentes e promover a vantagem competitiva, pois pode facilitar o acesso a recursos produtivos que sejam escassos.

O acesso aos recursos físicos, financeiros, organizacionais e humanos é fundamental para aumentar o desempenho da empresa. Os recursos são fonte de valor para a empresa e a relação entre o acesso aos recursos e o desempenho assume um papel central nas discussões acadêmicas e empresariais (ANDREASSI; SBRAGIA, 2002).

Uma vez que no Brasil existem muitas regiões industriais, proporcionando um campo fértil para pesquisas em aglomerações territoriais (ANDRADE; HOFFMANN, 2010), as empresas de diferentes portes estão em busca de novas formas para obter vantagem competitiva, para a manutenção e sobrevivência no mercado de atuação. Em função da globalização e suas implicações, muitas empresas estão optando e procurando inserir-se em aglomerações, para manter a competitividade com grandes empresas (LIMA; CARVALHO, 2011).

As pesquisas demonstram que as aglomerações de empresas que exercem determinada atividade econômica podem trazer vantagem competitiva para o setor em que atuam, além de impactar positivamente em setores correlatos, contribuindo para o desenvolvimento local e regional (GEROLAMO et al., 2005). A temática tem sido discutida e abordada em pesquisas nacionais e internacionais, especialmente pela existência de um número expressivo de regiões industriais espalhadas pelo país e pelo mundo.

Em seu estudo Li, Zubielqui e O'Connor (2015) constataram que as empresas aglomeradas podem gerar mais valor ao elevar ao máximo os vínculos externos com os recursos estratégicos, comparando com as empresas que não atuam em aglomeração. Da mesma forma, Gold, Seuring e Beske (2010) identificaram que em aglomerações, as relações são grandes oportunidades de adquirir recursos para as empresas.

Neste sentido, a aglomeração territorial exerce um papel importante no acesso aos recursos, contribuindo para um desempenho superior da empresa aglomerada em relação aos concorrentes dispersos geograficamente (EXPÓSITO-LANGA; TOMÁS-MIQUEL; MOLINA-MORALES, 2015). A comparação entre empresas de um mesmo segmento, dispersas e localizadas em aglomeração territorial, tem sido determinante para compreender o desempenho individual das empresas (MASCENA; FIGUEIREDO; BOAVENTURA, 2013).

Para Almeida e Silva (2015), com o processo de migração regional, diversas aglomerações têm surgido, favorecendo, inclusive, a realização de pesquisas acadêmicas sobre a temática. Essas pesquisas vêm conquistando maior destaque na Academia e na sociedade, em virtude do desenvolvimento local, regional e nacional, contribuindo, também, com o estabelecimento de novas relações interorganizacionais.

De acordo com Wilke e Diettrich (2020), com cenários cada vez mais incertos, as empresas precisam tomar decisões assertivas, utilizando de forma eficaz os recursos disponíveis, que podem auxiliar na sobrevivência e, além disso, gerar um desempenho 
superior aos seus concorrentes. Da mesma forma, Ribeiro et al. (2017) ressaltam um movimento intenso entre as empresas por uma melhor posição de mercado, identificando recursos que possibilitem aumentar o desempenho e a vantagem competitiva.

As empresas buscam desempenho acima da média, explorando recursos para criar vantagem competitiva (HITT; IRELAND; HOSKISSON, 2019). Com isso, identificar e analisar os recursos estratégicos pode aumentar a competitividade da empresa, a fim de desenvolver vantagem em relação aos concorrentes (GOULART; ZIBER, 2016).

Os estudos, no entanto, têm sido desenvolvidos em diversos setores e buscam investigar a relação entre os recursos e o desempenho, mas ainda não contemplam a análise dos diferentes tipos de recursos utilizados pelas empresas. Assim, tem-se como questão de pesquisa: Qual é o efeito do acesso aos recursos no desempenho das empresas, considerando os diferentes tipos de recursos?

Desta forma, o presente estudo tem como objetivo investigar o efeito do acesso aos recursos no desempenho de empresas do setor metalomecânico no contexto de aglomeração territorial. Com isso, o principal argumento da pesquisa está voltado ao acesso aos recursos e seu efeito no desempenho das empresas, a partir de um contexto de aglomeração territorial. A aglomeração pode facilitar o acesso aos diferentes tipos de recursos, gerando desempenho superior e, consequentemente, uma vantagem competitiva para as empresas aglomeradas.

\section{REFERENCIAL TEÓRICO}

A aglomeração territorial proporciona vantagem competitiva às empresas, pois possibilita acesso aos recursos, sejam eles físicos, financeiros, organizacionais ou humanos. $\mathrm{O}$ acesso aos recursos pode ser facilitado em um contexto de aglomeração territorial em virtude da proximidade geográfica e relacional entre as empresas, o que pode proporcionar um desempenho superior. Assim, a aglomeração territorial possibilita a facilidade de acesso aos recursos que influenciam o desempenho das empresas.

\section{Aglomeração Territorial}

Desde os ensaios teóricos de Marshall (1985), vários estudos empíricos detectaram que as empresas localizadas em aglomeração territorial apresentam desempenhos superiores em relação àquelas que estão dispersas geograficamente, embora pertençam ao mesmo setor (RUIZ-ORTEGA; PARRA-REQUENA; GARCÍA-VILLAVERDE, 2016). O fenômeno da aglomeração territorial é definido por Marshall (1985) como indústrias localizadas, ou seja, a concentração de empresas em uma mesma localidade, em que cada uma exerce um único ramo de produção ou apenas uma parte dele.

De forma semelhante, Porter (1999) define os aglomerados como empresas concentradas geograficamente, que estejam inter-relacionadas e ligadas por elementos em comum e subsídios complementares. Embora estejam próximas fisicamente, as empresas assumem formas diferenciadas, dependendo da natureza e particularidades de cada atividade que realizam. Desse modo, um aglomerado é composto por empresas que trabalham com produtos e serviços, fornecedores especializados, equipamentos e empresas de outros setores correlatos. Não obstante, as empresas que estão em aglomeração competem entre si, mas ao mesmo tempo cooperam entre si (PORTER, 1999). 
De outra forma, pode-se conceber que a aglomeração se trata de uma concentração de empresas com tamanhos e setores diversos (mas correlacionados) em uma determinada área geográfica (NEUMANN; HEXSEL; BALESTRIN, 2011; MARCO-LAJARA et al., 2016). As aglomerações podem ter diferenciações em relação a: sua história, contextos sociais, organização institucional, evolução e cultura. Ainda pode estar ligada à estrutura produtiva, formatos de governança, arranjo industrial, logística, associativismo, cooperação entre os atuantes, formatos de aprendizagem e, também, disseminação de conhecimento (SILVEIRA; DREHER; ULLRICH, 2005). Desse modo, o pressuposto básico da aglomeração territorial é a facilidade no acesso aos recursos, a exemplo das indústrias metalúrgicas da Inglaterra, que se estabeleceram nas proximidades das minas ou lugares com combustível barato (MARSHALL, 1985).

Neste contexto, existe uma grande diversidade de denominações sobre aglomeração de empresas, dificultando, assim, uma adequada classificação, o que impacta negativamente nas pesquisas acadêmicas e nas políticas públicas (SARACENI; ANDRADE Jr.; RESENDE, 2013). Nos estudos sobre o desenvolvimento local e regional a aglomeração pode receber diversas denominações, normalmente são identificadas como de cluster, distrito industrial, arranjo produtivo local (APL) e Sistemas Locais de Produção (SARACENI; ANDRADE Jr.; RESENDE, 2013).

Na literatura é possível identificar definições para a aglomeração, como cluster, APL e sistemas locais de produção e inovação (SLPIs), os quais indicam que é necessário, além da territorialização, condições específicas para cada denominação. Cada nomenclatura pode descrever distintas etapas do desenvolvimento da aglomeração de empresas, advertindo, desta forma, que a aglomeração de uma mesma cadeia produtiva em determinada região pode caracterizar-se de formas diferentes (LUBECK; WITTMANN; SILVA, 2012).

Para Pugas e Fernandes (2014) as definições mais utilizadas e apropriadas para definir um aglomerado são os distritos industriais, clusters e arranjos produtivos locais (APLs). Algumas definições estão voltadas ao tamanho da aglomeração, proximidade geográfica, tipo da empresa, setor, cooperação e competitividade no aglomerado. Independentemente da abordagem utilizada, no entanto, o que se torna essencial é o aproveitamento das interações e sinergias geradas pelas empresas do aglomerado, que podem auxiliar na obtenção de vantagem competitiva.

As aglomerações surgem em economias de desenvolvimento, podendo adotar formas distintas, de acordo com sua profundidade, temporalidade e sofisticação, podendo variar de dimensão, magnitude e estágio de desenvolvimento (CZAJKOWSKI; CUNHA, 2010). Essas aglomerações geralmente ocorrem pela busca das empresas em atuarem de forma cooperada, com o ideal de melhorar sua capacidade de competir, em relação com as empresas que atuam de forma individual na região, sendo compostas de micro, pequenas e médias empresas (BRITO et al., 2010).

Em se tratando de economias da aglomeração, podem ser caracterizadas como a concentração de atividades relacionadas, mas levando-se em conta determinados limites geográficos. A proximidade geográfica nessas atividades gera efeitos positivos para as empresas e colaboradores que se instalam em uma mesma região (DALBERTO; CIRINO; STADUTO, 2016). 
Pesquisas apontam, no entanto, que a aglomeração de empresas, vinculadas a uma mesma atividade econômica, pode resultar em efeitos positivos, mas também negativos, advindos da mão de obra especializada, infraestruturas novas e troca de conhecimentos. Estes fatores, positivos ou negativos, podem impactar diretamente no desempenho da empresa (THOMAZ et al., 2011). Para Brusco (1990), as aglomerações se caracterizam pela grande quantidade de organizações que se envolvem em processos de produção de um produto homogêneo e são demarcados pela demanda produtiva e sua capacidade.

De acordo com Hansen e Melo (2008), diversos estudos vêm destacando um conjunto de elementos e processos que são classificados como tácitos ou intangíveis, em que os intangíveis estão ligados à troca de informações, conhecimento e aprendizado, auxiliando em ações coletivas, que permitam que as empresas aglomeradas usem suas características para os processos de desenvolvimento econômico. Desta forma, a construção dos fatores intangíveis possibilita melhora no processo de inovação e maximização de recursos coletivos entre as empresas localizadas.

Não se pode ignorar, porém, que as empresas são diferentes umas das outras, e em virtude disto pode resultar em um mal-entendimento sobre as vantagens locais, advindas da aglomeração e como as organizações lidam com as vantagens. Com isso, o planejamento das políticas de desenvolvimento local podem priorizar outros fatores para esse grupo de empresas aglomeradas, que, deste modo, podem ser irrelevantes e prejudiciais para o grupo (TAVARES et al., 2015). Segundo Lemos (1997), as aglomerações possuem características específicas e que as distinguem umas das outras.

No decorrer dos anos diversas modificações industriais ocorreram nas organizações, exigindo adaptação e desenvolvimento de habilidades e competências. Uma dessas mudanças foi a relação entre as empresas, gerando a aproximação territorial, seja ela por meio de redes, arranjos produtivos locais, cluster ou aglomerações de empresas, estas aproximações criam oportunidade para o aumento do desempenho empresarial (CICONET; ANTUNES; RUFFONI, 2014).

\section{Recursos da empresa}

Os recursos da empresa podem ser fontes de vantagem competitiva (TEECE, 1982; BARNEY, 1986). Esse argumento teórico tem sua origem no trabalho seminal de Edith Penrose (1959), que concebeu a empresa como um conjunto mais amplo de recursos (WERNERFELT, 1984). A empresa pode ter abundância e/ou escassez de recursos, mas necessita investir continuamente na renovação e aquisição de recursos (TEECE, 1982), consequentemente precisa ter acesso a eles.

Neste sentido, entende-se por recurso qualquer coisa que possa ser considerada uma força ou fraqueza de uma empresa em um determinado momento, mais especificamente podem ser definidos como ativos tangíveis e intangíveis (WERNERFELT, 1984). Além disso, os recursos da empresa podem ser classificados em quatro categorias: recursos físicos, recursos financeiros, recursos humanos e recursos organizacionais (BARNEY; HESTERLY, 2004). 
Os recursos físicos envolvem os bens tangíveis, como prédios, instalações, móveis, máquinas e equipamentos, ao passo que os recursos financeiros envolvem outros tipos de bens tangíveis, como o dinheiro que financia as atividades da empresa e, inclusive, direitos relacionados a créditos junto aos clientes, bancos, fornecedores, etc. Por outro lado, os recursos humanos compreendem bens intangíveis de atributo individual, como experiência, conhecimento, confiança, relacionamento e práticas de recursos humanos (e.g., treinamento), ao passo que os recursos organizacionais compreendem bens intangíveis de atributo de grupos de pessoas, como sistemas formais ou informais de planejamento, coordenação e controle, reputação e cultura organizacional (BARNEY; HESTERLY, 2017).

A visão baseada em recursos da empresa sustenta-se em dois pressupostos fundamentais (BARNEY, 1991; PETERAF, 1993): (1) a heterogeneidade - os recursos podem ser diferentes entre as empresas, ou seja, as empresas podem ter diferentes recursos; (2) a imobilidade - as diferenças de recursos entre as empresas podem ser mantidas ao longo do tempo, ou seja, elas podem não conseguir equiparar-se em termos de recursos no decorrer do tempo.

Desta forma, os recursos da empresa são heterogêneos e imóveis, observando-se que as diferenças em termos de recursos podem ser duradouras e estáveis, não só por causa das barreiras de entrada em um setor, mas também porque os recursos podem ser idiossincráticos (BARNEY; HESTERLY, 2004). Por outro lado, a escassez de recursos leva a empresa a utilizar recursos inferiores (PETERAF, 1993), impactando na sua posição no mercado (WERNERFELT, 1984), pois quando a empresa não tem recursos suficientes não consegue atender às demandas de mercado (TEECE, 1982).

Esses pressupostos podem explicar por que algumas empresas superam outras dentro de um mesmo setor. De tal modo que as implicações dos dois pressupostos permitem evidenciar possível vantagem competitiva entre as empresas. Assim, uma empresa tem vantagem competitiva quando cria mais valor econômico do que seus concorrentes, ou seja, a empresa tem um desempenho superior em relação aos concorrentes (BARNEY; HESTERLY, 2017). Com isso, ela terá uma vantagem competitiva quando adotar uma estratégia que crie valor e que não seja instituída ao mesmo tempo pelos concorrentes atuais ou potenciais.

Os recursos da empresa que se constituem ou não em fontes de vantagem competitiva podem ser identificados por meio do modelo desenvolvido por Barney (1991), que classifica os recursos a partir de quatro atributos. Esses atributos são valor, raridade, imitabilidade e organização, conhecidos pelo acrônimo VRIO ou modelo VRIO (BARNEY; CLARK, 2007). Para identificar o recurso que se caracteriza como fonte de vantagem competitiva deve-se responder às questões relativas a cada um dos atributos (BARNEY; HESTERLY, 2017): (1) Valor: o recurso permite que a empresa explore uma oportunidade ambiental e/ou neutralize uma ameaça do ambiente?; (2) Raridade: o recurso é controlado atualmente apenas por um pequeno número de empresas concorrentes?; (3) Imitabilidade: as empresas sem o recurso enfrentam uma desvantagem de custo para obtê-lo ou desenvolvê-lo?; (4) Organização: as outras políticas e procedimentos da empresa estão organizados para dar suporte à exploração de seus recursos valiosos, raros e custosos para imitar? (Quadro 1). 
Quadro 1 - Critérios para identificação da vantagem competitiva (Modelo VRIO)

\begin{tabular}{|c|c|c|c|c|c|}
\hline Valioso & Raro & $\begin{array}{c}\text { Custoso } \\
\text { de imitar }\end{array}$ & $\begin{array}{c}\text { Explorado pela } \\
\text { empresa }\end{array}$ & Implicação competitiva & Desempenho \\
\hline Não & - & - & Não & $\begin{array}{c}\text { Desvantagem } \\
\text { competitiva }\end{array}$ & Abaixo do normal \\
\hline Sim & Não & - & - & $\begin{array}{c}\text { Paridade } \\
\text { competitiva }\end{array}$ & Normal \\
\hline $\operatorname{Sim}$ & $\operatorname{Sim}$ & Não & - & $\begin{array}{c}\text { Vantagem competitiva } \\
\text { temporária }\end{array}$ & Acima do normal \\
\hline $\operatorname{Sim}$ & $\operatorname{Sim}$ & Sim & Sim & $\begin{array}{c}\text { Vantagem competitiva } \\
\text { sustentável }\end{array}$ & Acima do normal \\
\hline
\end{tabular}

Fonte: Adaptado de BARNEY e WRIGHT (1998) e BARNEY e HESTERLY (2017).

Um recurso valioso, raro e difícil de imitar e bem explorado pela empresa terá como implicação uma vantagem competitiva se tiver um desempenho acima do normal, ou seja, um desempenho superior aos concorrentes (BULGACOV; ARREBOLA; GOMEL, 2012). Assim, o valor e a raridade estão ligados à vantagem competitiva, da mesma forma que a vantagem competitiva está ligada ao desempenho da empresa. Desse modo, se os concorrentes conseguem adquirir o mesmo recurso ou seu substituto do mesmo modo que a empresa que já o possui, e ainda obter por um mesmo custo, então os recursos não podem ser fonte de vantagem competitiva para nenhuma das empresas (LAIMER; MARTINS; LAIMER, 2006).

Por outro lado, a combinação de recursos pode resultar no desenvolvimento de novos recursos. Assim, a identificação de um recurso que seja fonte de vantagem competitiva não elimina a expectativa de que outro recurso possa também ser fonte de vantagem competitiva, pois o uso e a interação entre os recursos criam várias possibilidades de vantagem competitiva (CARVALHO; PRÉVOT; MACHADO, 2014).

A empresa pode adquirir novos recursos aproveitando que o estoque de recursos existente pode criar assimetria na competição por recursos (WERNERFELT, 2011), pois a aquisição e a acumulação de recursos (i.e., estoque de recursos) são fatores centrais na heterogeneidade e imobilidade de recursos entre as empresas (MARITAN; PETERAF, 2011), tendo em vista que as empresas com recursos heterogêneos ao adquirirem novos recursos ampliam sua heterogeneidade inicial com o decorrer do tempo (WERNERFELT, 2011).

Assim sendo, a contínua aquisição e acumulação de recursos e as novas combinações deles proporciona vantagem competitiva para a empresa e essa vantagem pode ser sustentável (PENROSE, 1959). O acesso aos recursos, nesse sentido, constitui-se como elemento central para criar e manter vantagem competitiva. $\mathrm{O}$ acesso aos recursos fornece condições para cria valor, impactando no desempenho da empresa e, consequentemente, podendo criar vantagem competitiva (BARALDI; GRESSETVOLD; HARRISON, 2012). 


\section{Desempenho}

Na literatura existem diversos métodos de mensuração do desempenho (BRITO; BRITO; MORGANTI, 2009). A mensuração do desempenho possibilita compreender se as metas e objetivos propostos estão sendo alcançados, com esse desempenho podendo ser mensurado por meio dos recursos estratégicos (BEUREN; MARCELLO, 2016). Além disso, a mensuração do desempenho das empresas pode estar relacionada com as vendas e as informações do lucro (HUNG et al., 2010).

$O$ desempenho também pode estar relacionado com a capacidade que as empresas têm em se adaptar às mudanças frequentes do mercado, ou seja, olhando para dentro da empresa (e.g., análise dos recursos internos), mas ao mesmo tempo para fora dela (e.g., análise de mercado), sabendo como lidar de forma ativa com as mudanças no mercado (SILVA, 2014).

Venkatraman e Ramanujam (1986) apontam que o desempenho pode ser medido por meio dos resultados financeiros, pois estes apresentam indicadores que demostram o desempenho da empresa em relação às metas estabelecidas, servindo de subsídio para a mensuração. Neste sentido, se a empresa obtiver o desempenho desejado, considera-se que os recursos possibilitam a vantagem competitiva em relação aos seus concorrentes.

A mensuração do desempenho é a maneira de entender se os objetivos desejados estão sendo alcançados, destacando-se que estes podem ser avaliados a partir dos recursos estratégicos das empresas, podendo ser de capital humano, estrutural ou físico (BEUREN; MARCELLO, 2016). O desempenho das empresas pode ser apurado pela capacidade de se adaptar às mudanças, porém é importante observar a capacidade de lidar com as mudanças do mercado (SILVA, 2014). As vendas e o lucro das empresas podem estar ligados às medidas de desempenho (HUNG et al., 2010).

$\mathrm{O}$ desempenho pode ser mensurado com medidas objetivas ou subjetivas. As medidas objetivas envolvem indicadores financeiros ou econômicos, ao passo que as medidas subjetivas abrangem a percepção dos proprietários e/ou gestores (VENKATRAMAN; RAMANUJAM, 1986). As medidas subjetivas têm recebido respaldo na literatura, pois as medidas objetivas somente estão disponíveis em empresas de capital aberto (i.e., empresas que possuem ações na Bolsa de Valores). Além disso, as medidas objetivas podem refletir o desempenho econômico ou financeiro, em que o indicador de participação de mercado da organização está ligado ao desempenho econômico. Já a rentabilidade e a margem de lucro estão relacionadas ao desempenho financeiro (WANG; AHMED, 2007). Desse modo, o desempenho da empresa é a medida que possibilita evidenciar a existência de vantagem competitiva (BARNEY; HESTERLY, 2017).

\section{Recursos e Desempenho}

Um contexto de aglomeração territorial produz sinergias e benefícios, resultantes das externalidades geradas em um espaço geográfico com concentração de empresas de um mesmo setor (MARCO-LAJARA et al., 2016). Assim, o pressuposto básico da aglomeração é a existência de economias externas que beneficiam as empresas devido a dotações de recursos (MARSHALL, 1985). As empresas beneficiam-se do acesso aos recursos físicos (e.g., insumos), recursos financeiros (e.g., linhas de crédito em institui- 
ções financeiras), recursos humanos (e.g., mão de obra qualificada) e recursos organizacionais (e.g., informações de entidades setoriais), além de uma atmosfera industrial, que se traduz em recursos intangíveis, baseados no compartilhamento de informações e conhecimento.

A proximidade geográfica e relacional que uma aglomeração territorial fornece é um elemento que auxilia as empresas a obter acesso especial a relacionamentos próximos, melhores informações, incentivos poderosos e outros benefícios difíceis de obter a distância (RUIZ-ORTEGA; PARRA-REQUENA; GARCÍA-VILLAVERDE, 2016). Isso permite que as empresas localizadas em aglomerações territoriais obtenham vantagens competitivas que se traduzem em um desempenho superior aos concorrentes.

Os recursos da empresa proporcionam vantagem competitiva a partir da criação de valor, que se traduz no desempenho superior aos concorrentes (BARNEY; HESTERLY, 2017). Com isso, o acesso aos recursos físicos, recursos financeiros, recursos humanos e recursos organizacionais pode fornecer condições para que a empresa tenha um desempenho superior aos concorrentes, posto que as empresas que possuem recursos que sejam fonte de vantagem competitiva conseguem obter desempenho acima da média dos concorrentes (HITT; IRELAND; HOSKISSON, 2019).

O acesso aos recursos físicos explica o desempenho das empresas (KARIAA; WONGB; ASAARIC, 2012). Aquelas que possuem facilidade de acesso a matérias-primas, máquinas e equipamentos, apresentaram uma relação positiva entre o acesso aos recursos físicos e o desempenho. Em outras palavras, pode-se afirmar que os recursos físicos ajudam a melhorar o desempenho da empresa (LAIMER; WEBBER; FERRON, 2016). Desse modo, com base nesses argumentos teóricos propõe-se a seguinte hipótese de pesquisa:

Hipótese $1\left(H_{1}\right)$ : O acesso aos recursos físicos tem efeito positivo no desempenho.

Da mesma forma, os recursos financeiros exercem a função de financiar as atividades operacionais da empresa, facilitando a criação e adoção de estratégias cujo objetivo visa a aumentar a rentabilidade, consequentemente pode resultar em um desempenho maior do que a média do setor (BARNEY; HESTERLY, 2017). De tal modo que as empresas estão sempre em busca da maior eficiência e de menores custos, observando-se que os recursos estratégicos auxiliam nessa busca, proporcionando melhor desempenho (BEUREN; MARCELLO, 2016). Com base nesses argumentos teóricos apresenta-se a seguinte hipótese de pesquisa:

Hipótese $2\left(\mathrm{H}_{2}\right)$ : $\mathrm{O}$ acesso aos recursos financeiros tem efeito positivo no desempenho.

Os recursos organizacionais consistem nas rotinas e processos internos da empresa, incluindo sistemas informatizados de controle e gestão, passando pela facilidade em identificar oportunidades, ameaças, pontos fortes e pontos fracos da empresa. Esses recursos exercem influência positiva no desempenho, pois as empresas que concentram mais esforços na sua organização interna, com a finalidade de aprimorar sua gestão e controle, conseguem melhores resultados (LAIMER; WEBBER; FERRON, 2016). 
O desempenho das empresas pode ser explicado com base no acesso aos recursos organizacionais (KARIAA; WONGB; ASAARIC, 2012). Os recursos organizacionais podem proporcionar aumento de produtividade, melhorando o desempenho da empresa (BARNEY; HESTERLY, 2017). Assim, esses argumentos teóricos permitem formular a seguinte hipótese de pesquisa:

\section{Hipótese $3\left(\mathrm{H}_{3}\right)$ : O acesso aos recursos organizacionais tem efeito positivo no} desempenho.

As práticas de recursos humanos estão significativamente relacionadas ao desempenho financeiro, o que corrobora os resultados de outros estudos (OGUNYOMI; BRUNING, 2016). Os recursos humanos são compostos por profissionais qualificados, que oferecem algum tipo de diferencial para as empresas, seja em termos de produtividade ou de redução de custos (LAIMER; WEBBER; FERRON, 2016).

Além disso, o desempenho das empresas também pode ser explicado pelo acesso a determinados tipos de recursos humanos (KARIAA; WONGB; ASAARIC, 2012). Os recursos humanos são parte da base de recursos que permite à empresa ganhar vantagem competitiva (BARNEY; HESTERLY, 2017). A partir desses argumentos teóricos elaborou-se a seguinte hipótese de pesquisa:

Hipótese $4\left(\mathrm{H}_{4}\right)$ : O acesso aos recursos humanos tem efeito positivo no desempenho.

Esses elementos permitem que empresas em um contexto de aglomeração territorial obtenham vantagens competitivas que se traduzem em desempenho superior (RUIZ-ORTEGA; PARRA-REQUENA; GARCÍA-VILLAVERDE, 2016). Os estudos seminais (e.g., PENROSE, 1959) fornecem uma explicação abrangente sobre a relação entre os recursos e o desempenho da empresa.

Desta forma, a empresa que investe continuamente na renovação de seus recursos por meio de novas combinações de recursos pode ter uma vantagem competitiva sustentável (PENROSE, 1959). Com isso, a facilidade de acesso aos recursos físicos, financeiros, organizacionais e humanos pode proporcionar um desempenho superior aos concorrentes, criando e mantendo uma vantagem competitiva. Assim, com base nos argumentos teóricos e nas hipóteses de pesquisa formulou-se um modelo teórico proposto (Figura 1), que busca investigar o efeito do acesso aos recursos no desempenho de empresas do setor metalo-mecânico no contexto de aglomeração territorial.

Figura 1 - Modelo teórico proposto

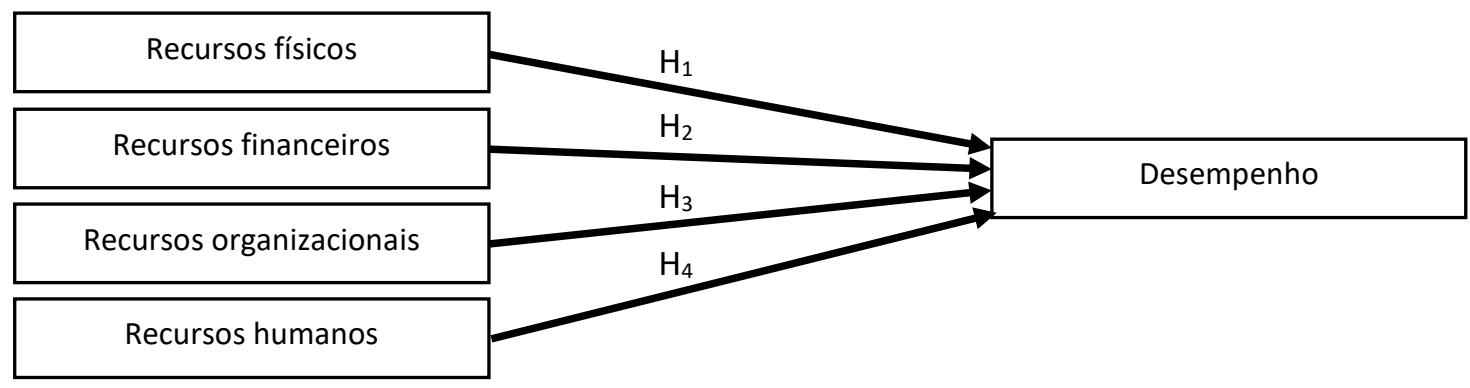

Fonte: Elaborado pelos autores (2020). 


\section{MÉTODO}

O estudo caracteriza-se como uma pesquisa quantitativa e descritiva, cujo propósito é estabelecer a relação entre as variáveis e testar as hipóteses formuladas. Nesse sentido, os procedimentos metodológicos foram baseados em uma survey, a partir de uma pesquisa de campo, com a coleta de dados de um conjunto de elementos da população.

Como critério de seleção considerou-se as empresas do setor metalomecânico, dos segmentos de fabricação de produtos de metal, fabricação de máquinas e equipamentos e metalúrgica. A seleção das empresas foi realizada por meio do site Empresas do Brasil, a partir da Classificação Nacional de Atividade Econômica - Cnae. Assim, identificaram-se as empresas do setor e dos segmentos escolhidos que estavam localizadas no município de Passo Fundo, situado no norte do Estado do Rio Grande do Sul.

A pesquisa utilizou a técnica de amostragem por conveniência, uma técnica não probabilística, em que a escolha das empresas que participaram da pesquisa considerou a proximidade geográfica das empresas em relação aos pesquisadores. A amostra obtida resultou em 73 empresas que se disponibilizaram e participaram da pesquisa.

Desta forma, optou-se pelo setor metalomecânico como objeto de análise, por ser um grande gerador de empregos, pelo número expressivo de empresas e sua importância para a economia do Estado do Rio Grande do Sul. De acordo com Souza (2006), a partir dos anos 90 o desempenho do setor tem evoluído consideravelmente no Brasil e em seus diferentes Estados, especialmente, no Rio Grande do Sul.

A técnica de coleta de dados utilizada nesse estudo foi uma survey, sendo realizada por meio de um questionário semiestruturado, desenvolvido com base na literatura sobre recursos e desempenho. Os constructos foram compostos por uma escala Likert de 11 pontos variando de 0 (discordo totalmente) a 10 (concordo totalmente) (Quadro 2). Para preenchimento dos questionários foram levados em consideração os sujeitos, ocupantes de cargos de gestão ou coordenação das empresas pesquisadas. A aplicação dos questionários foi realizada de duas maneiras, presencialmente e via e-mail; quando utilizado da forma presencial foi feito contato telefônico com as empresas e agendado um horário com o responsável. Já os questionários via e-mail foram enviados para o responsável, o qual foi contatado também via telefone para explicar e auxiliar o respondente.

O questionário foi validado por três experts da área. Após a validação e as correções sugeridas para melhoria do questionário, este foi submetido a um pré-teste, com três empresas do setor pesquisado, para ser possível verificar a clareza das questões, se a interpretação do respondente corresponde ao significado esperado para a pesquisa e o tempo necessário para o preenchimento do questionário.

Para análise e interpretação dos resultados foi necessária a realização de análises estatísticas, quando foi utilizado o programa SPSS Statistics 21. Para análise de dados foi utilizada a estatística inferencial (regressão linear múltipla), buscando mensurar e analisar as relações entre as variáveis dependentes e independentes. 
Quadro 2 - Constructos e variáveis utilizadas para construção do questionário

\begin{tabular}{|c|c|c|c|c|}
\hline Constructos & Variáveis estudadas & Item & Tipo de questão & Literatura \\
\hline $\begin{array}{c}\text { Caracterização da } \\
\text { empresa }\end{array}$ & $\begin{array}{c}\text { Nome da empresa } \\
\text { Município } \\
\text { Segmento } \\
\text { Ano de início } \\
\text { Número de empregados } \\
\text { Porte (faixas de faturamento) }\end{array}$ & 7 & $\begin{array}{c}\text { Aberta e de } \\
\text { múltipla escolha }\end{array}$ & $\begin{array}{c}\text { Elaborado pelos } \\
\text { autores }\end{array}$ \\
\hline Recursos & $\begin{array}{c}\text { Recursos físicos } \\
\text { Recursos financeiros } \\
\text { Recursos organizacionais } \\
\text { Recursos humanos }\end{array}$ & 16 & $\begin{array}{l}\text { Escala de } 11 \\
\text { pontos }\end{array}$ & $\begin{array}{l}\text { Barney e Hesterly } \\
\text { (2017) }\end{array}$ \\
\hline Desempenho & $\begin{array}{c}\text { Faturamento } \\
\text { Aumento de vendas } \\
\text { Redução de custos } \\
\text { Aumento da rentabilidade } \\
\text { Aumento da produtividade } \\
\text { Aumento do lucro } \\
\end{array}$ & 6 & $\begin{array}{l}\text { Escala de } 11 \\
\text { pontos }\end{array}$ & $\begin{array}{c}\text { Venkatraman e } \\
\text { Ramanujam (1986); } \\
\text { Wang e Ahmed (2007) } \\
\text { Hung et al. (2010); } \\
\text { Beuren e Marcello, } \\
\text { 2016. } \\
\end{array}$ \\
\hline
\end{tabular}

Fonte: Elaborado pelos autores (2020).

Para a análise dos dados foram verificados os dados discrepantes ou com falta de respostas, os quais foram eliminados da análise. Após realizada a tabulação dos dados foi verificada a normalidade dos dados por meio da assimetria e curtose, sendo que as variáveis preencheram os requisitos de normalidade. Na sequência foi realizado o teste de confiabilidade das escalas dos construtos Recursos Físicos, Recursos Financeiros, Recursos Organizacionais, Recursos Humanos e Desempenho, observando-se que o coeficiente Alfa de Cronbach ( $\alpha>0,600)$ foi considerado aceitável (MALHOTRA, 2012).

Após a realização dos testes que servem de pré-requisito, foi utilizada a técnica de análise de regressão linear para verificar a relação entre os constructos. Assim, foi utilizada a regressão linear múltipla, buscando mensurar e analisar as relações entre as variáveis dependente e independentes, sendo no caso deste estudo um único modelo de regressão linear (Quadro 3).

Quadro 3 - Modelo de regressão linear múltipla proposto

\begin{tabular}{|c|c|c|c|}
\hline Modelo & Variável Dependente & Variável Independente & Hipótese \\
\hline \multirow{3}{*}{$\begin{array}{c}\text { Regressão } \\
\text { Linear } \\
\text { Múltipla }\end{array}$} & \multirow{3}{*}{ Desempenho } & Recursos Físicos & Hipótese 1 \\
\cline { 2 - 3 } & & Recursos Financeiros & Hipótese 2 \\
\cline { 2 - 3 } & & Recursos Organizacionais & Hipótese 3 \\
\cline { 2 - 3 } & & Recursos Humanos & Hipótese 4 \\
\hline
\end{tabular}

Fonte: Elaborado pelos autores (2020).

\section{RESULTADOS E DISCUSSÃO}

\section{Características das Empresas Pesquisadas}

As 73 empresas pesquisadas do setor metalomecânico foram classificadas observando-se a sua idade, porte, número de empregados e o segmento em que atuam. Percebe-se em relação à idade da amostra pesquisada, que a tendência central da amostra, 
ou seja, que $50 \%$ das empresas têm idade igual a 10 anos ou menos, $25 \%$ têm idade igual ou menor que 5,5 anos, e $25 \%$ da amostra têm em torno de 24 anos de idade ou menos.

Em relação ao número de empregados e porte das empresas, classificou-se da seguinte forma: microempreendedor individual 0 a 1 empregado, faturamento inferior a $\mathrm{R} \$ 81$ mil; microempresa de 2 a 9 empregados, faturamento inferior a $\mathrm{R} \$ 360 \mathrm{mil}$; pequena empresa de 10 a 49 empregados, faturamento entre $R \$ 360$ mil a $R \$ 4,8$ milhões; média empresa de 50 a 99 empregados, faturamento entre $R \$ 4,8$ milhões a $R \$ 300$ milhões, e grande empresa mais de cem empregados e um faturamento superior a $\mathrm{R} \$$ 300 milhões.

Das empresas pesquisadas a maioria está classificada como microempresa, 39,7\%, com 2 a 9 empregados; em segundo lugar encontra-se o microempreendedor individual com 28,8\%, entre 0 a 1 empregado. Assim, percebe-se que a maior concentração de empresas pesquisadas está entre essas duas classificações, que no total representam $68,5 \%$ da amostra. As empresas de pequeno, médio e grande porte representam $31,5 \%$ da amostra. Quanto ao número de empregados, a maioria das empresas possui de 0 a 9 empregados, representando $80,82 \%$ da amostra.

A amostra foi dividida em três segmentos, fabricação de máquinas e equipamentos agrícolas, fabricação de produtos de metal e metalúrgica. O segmento com maior representatividade foi a fabricação de produtos de metal, $61,6 \%$, ou seja, mais da metade da amostra; em seguida a metalúrgica, com $23,3 \%$, e por fim a fabricação de máquinas e equipamentos agrícolas, com $15,1 \%$.

\section{Análise e Interpretação dos Resultados}

A partir da matriz de correlação de Pearson (Tabela 1) é possível perceber que não há colinearidade (multicolinearidade) entre as variáveis, ou seja, correlação forte superior a 0,80 ou 0,90 entre dois ou mais constructos. As correlações foram todas significativas no nível de 0,01 , além disso, todas as correlações são positivas, caminham no mesmo sentido, atendendo, assim, aos requisitos para o teste de regressão linear.

Tabela 1 - Matriz de correlação de Pearson

\begin{tabular}{lccccc}
\hline \multicolumn{1}{c}{ Variável } & Rec. Fis. & Rec. Fin. & Rec. Org. & Rec. Hum. & Desempenho \\
\hline Recursos físicos & 1,000 & & & & \\
Recursos financeiros & $0,656^{*}$ & 1,000 & & & \\
Recursos organizacionais & $0,537^{*}$ & $0,460^{*}$ & 1,000 & & \\
Recursos humanos & $0,698^{*}$ & $0,670^{*}$ & $0,442^{*}$ & 1,000 & \\
Desempenho & $0,690^{*}$ & $0,513^{*}$ & $0,681^{*}$ & $0,545^{*}$ & 1,000 \\
\hline
\end{tabular}

Nota: As correlações são significativas no nível de $p<0,01$.

Fonte: Elaborada pelos autores (2020)

Na sequência foram realizadas as análises de regressão linear múltipla, com o objetivo de investigar o efeito do acesso aos recursos (físicos, financeiros, organizacionais e humanos) no desempenho. A partir disso foi realizado o teste de significância do modelo, para verificar a significância da equação global proposta, constatando-se que o modelo foi significativo ao nível de $1 \%$ (Tabela 2). 
Tabela 2 - Análise de variância do modelo de regressão

\begin{tabular}{cccccccc}
\hline $\begin{array}{c}\text { Variável } \\
\text { Dependente }\end{array}$ & $\begin{array}{c}\text { Variáveis } \\
\text { Independentes }\end{array}$ & Item & $\begin{array}{c}\text { Soma dos } \\
\text { Quadrados }\end{array}$ & $\begin{array}{c}\text { Graus de } \\
\text { Liberdade }\end{array}$ & $\begin{array}{c}\text { Quadrado } \\
\text { Médio }\end{array}$ & F & Sig. \\
\hline Desempenho & Rec. Fís. & Regressão & 143,619 & 4 & 35,905 & 27,035 & 0,000 \\
& Rec. Fin. & Resíduos & 90,308 & 68 & 1,328 & & \\
& Rec. Org. & Total & 233,927 & 72 & & & \\
& Rec. Hum. & & & & & & \\
\hline
\end{tabular}

Fonte: Elaborada pelos autores (2020).

0 modelo de regressão foi significativo $(p<0,05)$. Assim, existe efeito do acesso aos recursos no desempenho das empresas. Na Tabela 3 encontram-se os resultados dos coeficientes de determinação $\left(R^{2}\right)$.

Tabela 3 - Modelo de regressão linear múltipla

\begin{tabular}{ccccccc}
\hline Modelo & $\begin{array}{c}\text { Variável } \\
\text { Dependente }\end{array}$ & $\begin{array}{c}\text { Variáveis } \\
\text { Independentes }\end{array}$ & $R$ & $R^{2}$ & $R^{2}$ ajustado & $\begin{array}{c}\text { Erro padrão da } \\
\text { estimativa }\end{array}$ \\
\hline Rec. Fís. \\
Desempenho & $\begin{array}{c}\text { Rec. Fin. } \\
\text { Rec. Org. } \\
\text { Rec. Hum. }\end{array}$ & 0,784 & 0,614 & 0,591 & 1,15241 \\
& & & & \\
\hline
\end{tabular}

Fonte: Elaborada pelos autores (2020).

Para a análise do modelo estudado foi utilizado o valor de $\mathrm{R}^{2}$ para a regressão linear múltipla. $O$ poder de explicação foi de $61,4 \%$, ao passo que o $R^{2}$ ajustado foi de $59,1 \%$, sendo um valor significativo, uma vez que envolve os recursos físicos, financeiros, organizacionais e humanos como variáveis independentes e o desempenho como variável dependente. Em seguida foi realizado o teste de significância dos coeficientes de regressão parciais com as variáveis independentes (Tabela 4).

Tabela 4 - Teste de significância dos coeficientes de regressão

\begin{tabular}{|c|c|c|c|c|c|}
\hline Modelo & $\begin{array}{c}\text { Variável } \\
\text { Dependente }\end{array}$ & $\begin{array}{c}\text { Variável } \\
\text { Independente }\end{array}$ & $\begin{array}{c}\text { Coeficiente de regressão } \\
\text { parcial (Beta não } \\
\text { padronizado) }\end{array}$ & $\mathrm{T}$ & Sig. \\
\hline & & Constante & 0,488 & 0,763 & 0,448 \\
\hline & Desempenho & Rec. Fís. & 0,327 & 3,503 & 0,001 \\
\hline & & Rec. Fin. & $-0,001$ & $-0,016$ & 0,987 \\
\hline & & Rec. Org. & 0,473 & 4,735 & 0,000 \\
\hline & & Rec. Hum. & 0,046 & 0,597 & 0,553 \\
\hline
\end{tabular}

Fonte: Elaborada pelos autores (2020).

Na Tabela 4 está a análise do modelo, desempenho e recursos (físicos, financeiros, organizacionais e humanos). Este modelo busca investigar o efeito do acesso aos recursos no desempenho das empresas. Assim, a partir do modelo, nota-se que os recursos físicos e os recursos organizacionais têm efeito positivo e significativo $(p<0,01)$ no desempenho das empresas. Por outro lado, os recursos financeiros e os recursos humanos não apresentaram um efeito no desempenho das empresas.

Desta forma, a hipótese $\mathrm{H}_{1}-\mathrm{O}$ acesso aos recursos físicos tem efeito positivo no desempenho e a Hipótese $\mathrm{H}_{3}-\mathrm{O}$ acesso aos recursos organizacionais tem efeito positivo no desempenho foram confirmadas. Essas hipóteses corroboram a suposição de que 
o acesso aos recursos influencia o desempenho das empresas, baseado nos argumentos teóricos apresentados por alguns estudos (BARNEY; HESTERLY, 2017; HITT; IRELAND; HOSKISSON, 2019).

Por outro lado, a hipótese $\mathrm{H}_{2}$ - $\mathrm{O}$ acesso aos recursos financeiros tem efeito positivo no desempenho e a hipótese $\mathrm{H}_{4}$ - $\mathrm{O}$ acesso aos recursos humanos tem efeito positivo no desempenho, foram rejeitadas. Esperava-se que essas hipóteses confirmassem a suposição de que o acesso aos recursos financeiros e aos recursos humanos influencia o desempenho das empresas. Esses resultados são diferentes daqueles encontrados em outros estudos que tratam dos recursos financeiros (BEUREN; MARCELLO, 2016; BARNEY; HESTERLY, 2017) e dos recursos humanos (KARIAA; WONGB; ASAARIC, 2012; LAIMER; WEBBER; FERRON, 2016; OGUNYOMI; BRUNING, 2016).

Na Figura 2 apresenta-se o modelo teórico da pesquisa com os resultados da análise de regressão das variáveis recursos (físicos, financeiros, organizacionais e humanos) e desempenho.

Figura 2 - Modelo teórico da pesquisa

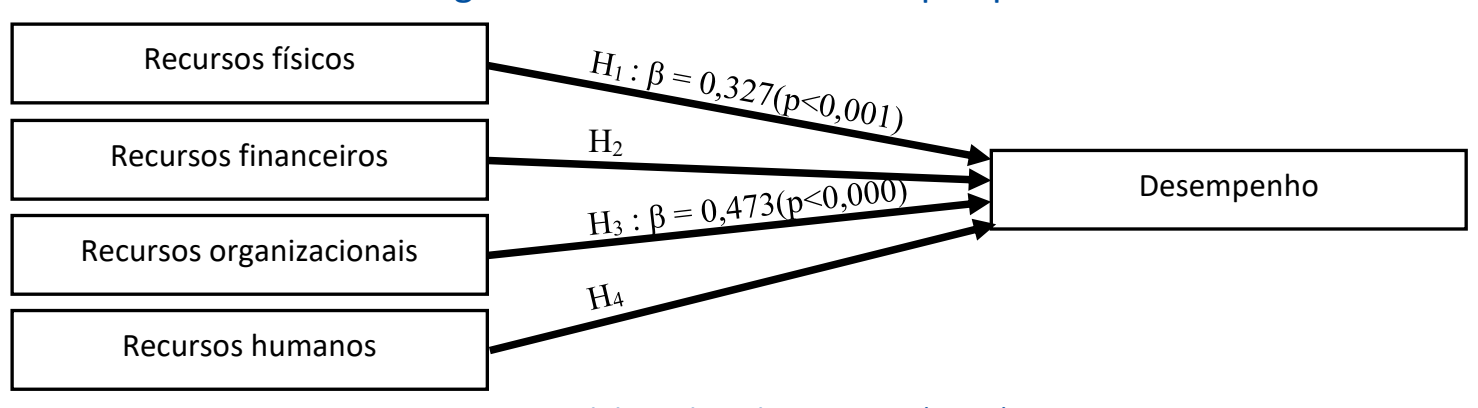

Fonte: Elaborada pelos autores (2020).

Os resultados encontrados indicam que o acesso aos recursos físicos e recursos organizacionais exercem influência no desempenho das empresas, ao passo que o acesso aos recursos financeiros e recursos humanos não evidenciaram influência no desempenho

De acordo com a literatura, os recursos da empresa explicam o seu desempenho (e.g., KARIAA; WONGB; ASAARIC, 2012). Quando a empresa tem acesso a matérias-primas, máquinas e equipamentos, pode aproveitar as oportunidades de mercado, atendendo às demandas, atingindo os objetivos estratégicos, como aumento da rentabilidade (LAIMER; WEBBER; FERRON, 2016).

Da mesma forma, os processos internos e os sistemas de planejamento, coordenação e controle possibilitam a criação e estabelecimento de estratégias que promovem o aumento de produtividade, melhorando o desempenho da empresa (BARNEY; HESTERLY, 2017). Esse desempenho da empresa pode ser explicado com base no acesso aos recursos organizacionais (KARIAA; WONGB; ASAARIC, 2012). Assim, os recursos exercem influência positiva no desempenho da empresa, pois aquelas que concentram mais esforços na sua organização interna, com a finalidade de aprimorar sua gestão e controle, conseguem melhores resultados (LAIMER; WEBBER; FERRON, 2016).

De modo geral, este estudo corrobora com os estudos de outros pesquisadores (LAIMER; LAIMER, 2009). Além disso, investigar o desempenho das empresas tem sido abordado por outros trabalhos (BRITO; BRITO; MORGANTI, 2009; GRONUM; VERREYN- 
NE; KASTELLE, 2012). Nesse sentido, os recursos das empresas em um contexto de aglomeração territorial podem ser determinantes para obter um desempenho superior (RUIZ-ORTEGA; PARRA-REQUENA; GARCÍA-VILLAVERDE, 2016).

\section{CONCLUSÃO}

Este estudo buscou investigar o efeito do acesso aos recursos no desempenho das empresas do setor metalomecânico no contexto de aglomeração territorial. A argumentação teórica está embasada na literatura sobre recursos e desempenho. $\mathrm{O}$ acesso aos recursos estratégicos, sejam eles físicos, financeiros, organizacionais ou humanos, pode influenciar o desempenho nas empresas, aumentando sua vantagem competitiva.

$\mathrm{Na}$ relação entre recursos como variável preditora do desempenho, os recursos físicos e organizacionais influenciam positivamente no desempenho nas empresas pesquisadas, sendo seus resultados significativos e positivos, ao passo que os recursos financeiros e humanos não exercem influência no seu desempenho.

Por meio destes resultados, pode-se afirmar que as hipóteses, 1 e 3 foram aceitas e as hipótese 2 e 4 foram rejeitadas, condizendo com outras pesquisas, relacionadas aos recursos e desempenho. Percebe-se a partir dos resultados encontrados que o acesso aos recursos físicos e organizacionais são fatores importantes para melhorar o desempenho das empresas do setor metalomecânico e para o alcance da vantagem competitiva. Desse modo, é fundamental que as empresas aglomeradas territorialmente na cidade de Passo Fundo, norte do Rio Grande do Sul, invistam em recursos físicos, uma vez que as empresas pesquisadas são fábricas e necessitam de matéria-prima, máquinas e equipamento e, também, invistam em recursos organizacionais para ter a capacidade de atender às demandas de mercado.

O estudo contribui com a literatura, evidenciando que a facilidade de acesso aos recursos pode proporcionar um desempenho superior e, também, indicando que os recursos apresentam diferentes tipos de relação com o desempenho. O estudo tem implicações gerenciais - possibilita que os gestores identifiquem os recursos que podem ser fonte de vantagem competitiva - e governamentais - proporciona subsídios aos governantes na elaboração de políticas públicas, priorizando estímulos ou incentivos aos tipos de recursos que possam proporcionar um aumento na competitividade das empresas.

Deste modo, a pesquisa também contribui para o aumento de estudos sobre as temáticas em aglomerações ou concentrações de empresas brasileiras, somando-se às pesquisas quantitativas, que possuem poucos estudos sobre o tema. Assim, o acesso aos recursos proporcionará um maior desempenho nas empresas aglomeradas. Embora o estudo tenha seguido os padrões metodológicos, ele apresenta algumas limitações de pesquisa, como o tamanho da amostra, devido à dificuldade no acesso às informações e indisponibilidade dos gestores em responder o questionário.

Como sugestão de estudo futuros pode-se realizar pesquisas, visando à diferença entre os segmentos, fabricação de máquinas e equipamentos agrícolas, fabricação de produtos de metal e metalúrgica, em relação aos recursos, inovação e desempenho da empresa. Outra sugestão é utilizar como amostra empresas aglomeradas e não aglomeradas, fazendo assim uma comparação entre elas, verificando a existência das di- 
ferenças entre as empresas quanto ao acesso aos recursos, inovação e desempenho. Também pode-se utilizar a idade e tamanho das empresas como variáveis mediadoras para verificar eventuais diferenças entre as empresas aglomeradas e não aglomeradas.

\section{AGRADECIMENTO}

\section{Os autores agradecem o apoio financeiro da Fundação Meridional.}

\section{REFERÊNCIAS}

ALMEIDA, F. A.; SILVA, A. S. B. Índices de concentração: evidências empíricas à indústria de transformação cearense. In: ENCONTRO NACIONAL DE ENGENHARIA DE PRODUÇÃO, 35., 2015, Fortaleza. Anais [...]. Fortaleza: Enegep, 2015.

ANDRADE, M. A. R.; HOFFMANN, V. E. Redes interorganizacionais: um estudo das pequenas e médias empresas no setor calçadista do Vale do Rio Tijucas. Revista de Administração e Inovação, v. 7, n. 2, p. 193-216, 2010.

ANDREASSI, T.; SBRAGIA, R. Relações entre indicadores de P\&D e de resultado empresarial. Revista de Administração, v. 37, n. 1, p. 72-84, 2002.

BARALDI, E.; GRESSETVOLD, E.; HARRISON, D. Resource interaction in inter-organizational networks: Foundations, comparison, and a research agenda. Journal of Business Research, v. 65, n. 2, p. 266-276, 2012. BARNEY, J. B. Firm resources and sustained competitive advantage. Journal of Management, v. 17, n. 1, p. 99-120, 1991.

BARNEY, J. B. Strategic Factor Markets: Expectations, Luck, and Business Strategy. Management Science, v. 32, n. 10, p. 1.231-1.241, 1986.

BARNEY, J. B.; CLARK, D. N. Resource-based theory: creating and sustaining competitive advantage. Oxford: Oxford University Press, 2007.

BARNEY, J. B.; HESTERLY, W. Economia das organizações: entendendo a relação entre as organizações e a análise econômica. In: CLEGG, S. R.; Hardy, C.; NORD, W. R. (org.). Handbook de estudos organizacionais. São Paulo: Atlas, 2004. p. 131-179. V. 3.

BARNEY, J. B.; HESTERLY, W. S. Administração estratégica e vantagem competitiva: conceitos e casos. São Paulo: Pearson, 2017.

BARNEY, J. B.; WRIGHT, P. M. On becoming a strategic partner: The role of human resources in gaining competitive advantage. Human Resource Management, v. 37, n. 1, p. 31-46, 1998.

BEUREN, I. M.; MARCELLO, I. E. Relação da importância dos recursos estratégicos com as medidas de desempenho em empresas brasileiras. Revista Ibero-Americana de Estratégia, v. 15, n. 1, p. 64-82, 2016.

BRITO, E. P. Z.; BRITO, L. A. L.; MORGANTI, F. Inovação e o desempenho empresarial: lucro ou crescimento? Revista de Administração de Empresas, v. 8, n. 1, 2009.

BRITO, E. P. Z.; BRITO, L. A. L.; PORTO, C. E.; SZILAGYI, E. M. A relação entre aglomeração produtiva e crescimento: a aplicação de um modelo multinível ao setor industrial paulista. Revista de Administração Contemporânea, v. 14, n. 4, p. 615-632, 2010.

BRUSCO, S. The idea of the industrial district: its genesis. In: PYKE, F.; BECATTINI, G.; SENGENBERGER, W. Industrial districts and inter-firm co-operation in Italy. International Institute for Labour Studies, 1990.

BULGACOV, S.; ARREBOLA, M. C.; GOMEL, M. M. Recursos compartilhados: uma aplicação da visão baseada em recursos em um condomínio tecnológico no Paraná. Revista de Ciências da Administração, v. 14, n. 32, p. 92-106, 2012.

CARVALHO, D. M.; PRÉVOT, F.; MACHADO, J. A. D. O uso da teoria da visão em recursos em propriedades rurais: uma revisão sistemática da literatura. Revista de Administração, v. 49, n. 3, p. 506-518, 2014.

CICONET, I.; ANTUNES, J. A. V.; RUFFONI, J. Características das atividades para a geração da inovação no arranjo produtivo local metalmecânico automotivo da região de Caxias do Sul (RS). Revista Brasileira de Gestão e Inovação, v. 1, n. 3, p. 1-26, 2014.

CZAJKOWSKI, A.; CUNHA, S. K. Organização e coordenação da rede de cooperação em aglomerados de turismo rural. Turismo: Visão e Ação, v. 12, n. 1, p. 92-113, 2010.

DALBERTO, C. R.; CIRINO, J. F.; STADUTO, J. A. R. Especialização versus diversificação: economias de aglomeração e seus impactos sobre os salários industriais em Minas Gerais. Gestão \& Regionalidade, v. 32, n. 95, p. 143-159, 2016. 
EXPÓSITO-LANGA, M.; TOMÁS-MIQUEL, J. V.; MOLINA-MORALES, F. X. Innovation in clusters: exploration capacity, networking intensity and external resources. Journal of Organizational Change Management, v. 28, n. 1, p. 26-42, 2015.

GEROLAMO, C. M.; CARDOZA, E.; VAICARI, F.; CARPINETTI, R. C. L. Aglomeração de pequenas e médias empresa como ambiente propício à melhoria de desempenho - caracterização do arranjo produtivo local do setor de confecção de bordados de Ibitinga. Rev. Cent. Ciênc. Admin., Fortaleza, v. 11, n. 1, p. 82-92, 2005.

GOLD, S.; SEURING, S.; BESKE, P. Sustainable supply chain management and inter-organizational resources: a literature review. Corporate Social Responsibility and Environmental Management, v. 17, n. 1, p. 230-245, 2010.

GOULART, F. D.; ZIBER, A. M. A liderança setorial na cadeia do biodiesel: uma análise à luz do conceito de vantagem competitiva. Organizações Rurais \& Agroindustriais, v. 18, n. 4, p. 332-341, 2016.

GRONUM, S.; VERRENNE, M. L.; KASTELLE, T. The role of networks in small and medium-sized enterprise innovation and firm performance. Journal of Small Business Management, v. 50, n. 2, p. 257-282, 2012.

HANSEN, L. D.; MELO, L. O. R. Aglomeração de micro e pequenas empresas no Nordeste brasileiro. Revista Ciência e Administração, v. 14, n. 2, p. 304-323, 2008.

HITT, M. A.; IRELAND, R. D.; HOSKISSON, R. E. Administração estratégica: competitividade e globalização. São Paulo: Cengage Learning, 2019.

HUNG, R. Y. Y.; YANG, B.; LIEN, B. Y.; MCLEAN, G. N.; KUO, Y. Dynamic capability: Impact of process alignment and organizational learning culture on performance. Journal of World Business, v. 45, n. 3, p. 285294, 2010.

KARIAA, N.; WONGB, C. Y.; ASAARIC, M. H. A. H. Typology of resources and capabilities for firms' performance. Procedia-Social and Behavioral Sciences, v. 65, p. 711-716, 2012.

LAIMER, C. G.; LAIMER, V. R. Relações de cooperação na perspectiva da visão baseada em recursos. Revista de Administração da Unimep, 7 (3), p. 93-110, 2009.

LAIMER, C. G.; MARTINS, E. S.; LAIMER, V. R. A visão baseada em recursos como modelo para a vantagem competitiva sustentável: um estudo de caso. Revista de Administração e Ciências Contábeis do IDEAU, v. 1, n. 1, p. 27-40, 2006.

LAIMER, C. G.; WEBBER, C. C.; FERRON, F. Recursos y desempeño en las empresas brasileñas de servicios. In: ASAMBLEA ANUAL DEL CLADEA - CONSEJO LATINOAMERICANO DE ESCUELAS DE ADMINISTRACIÓN, 51., 2016, Medellín (Colômbia). Memorias [...]. Bogotá (Colômbia): Ascolfa, 2016. V. 1.

LEMOS, C. Notas preliminares do projeto arranjos locais e capacidade inovativa em contexto crescentemente globalizado. Rio de Janeiro: Mimeo, 1997.

LI, H.; ZUBIELQUI, G. C.; O'CONNOR, A. Entrepreneurial networking capacity of cluster firms: a social network perspective on how shared resources enhance firm performance. Small Business Economics, v. 45, n. 3, p. 523-541, 2015.

LIMA, G. B.; CARVALHO, D. T. Aglomerações industriais na cadeia têxtil: observações sobre a internacionalização do pólo TEC TEX. Revista Brasileira de Estratégia, v. 4, n. 2, p. 169-180, 2011.

LUBECK, M. R.; WITTMANN, L. M.; SILVA, S. M. A final, quais variáveis caracterizam a existência de cluster arranjos produtivos locais (APLS) e dos sistemas locais de produção e inovação (SLPIs). Revista Ibero-Americana de Estratégia, v. 11, n. 1, p. 120-151, 2012.

MALHOTRA, N. K. Pesquisa de marketing: uma orientação aplicada. 6. ed. Porto Alegre: Bookman, 2012. MARCO-LAJARA, B.; CLAVER-CORTÉS, E.; ÚBEDA-GARCÍA, M.; ZARAGOZA-SÁES, P. C. A dynamic analysis of the agglomeration and performance relationship. Journal of Business Research, v. 69, n. 5, p. 1.874-1.879, 2016.

MARITAN, C.; PETERAF, M. A. Building a bridge between resource acquisition and resource accumulation. Journal of Management, v. 37, n. 5, p. 1.374-1.389, 2011.

MARSHALL, A. Princípios de economia. 2. ed. São Paulo: Nova Cultura, 1985.

MASCENA, K. M. C.; FIGUEIREDO, F. C.; BOAVENTURA, J. M. G. Clusters e APL's: análise bibliométrica das publicações nacionais no período de 2000 a 2011. Revista de Administração de Empresas, v. 53, n. 5, p. 454-468, 2013.

NEUMANN, L.; HEXSEL, A.; BALESTRIN, A. Desafios à cooperação em aglomerados produtivos: um estudo de caso no segmento de malhas do sul do Brasil. Revista de Administração e Contabilidade da Unisinos, v. 8, n. 3, p. 220-230, 2011.

OGUNYOMI, P.; BRUNING, N. S. Human resource management and organizational performance of small and medium enterprises (SMEs) in Nigeria. The International Journal of Human Resource Management, $v$. 27, n. 6, p. 612-634, 2016. 
PENROSE, E. The theory of the growth of the firm. Oxford: Oxford University Press, 1959.

PETERAF, M. A. The cornerstones of competitive advantage: a resource-based view. Strategic Management Journal, v. 14, p. 3, p. 179-191, 1993.

PORTER, M. E. Competição: estratégias competitivas essenciais. 13. ed. Rio de Janeiro: Elsevier, 1999.

PUGAS, P. G. O.; FERNANDES, R. S. Externalidades positivas em aglomerações de empresas: um estudo no aglomerado de fundição da cidade de Cláudio (MG), Brasil. Gestão \& Regionalidade, v. 30, n. 89, p. 49-62, 2014.

RIBEIRO, J. S. A. N.; SOARES, M. A. C.; JURZA, P. H.; ZIVIANI, F.; NEVES, J. T. R. Gestão do conhecimento e desempenho organizacional: integração dinâmica entre competências e recursos. Perspectivas em Gestão e Conhecimento, v. 7, número especial, p. 4-17, 2017.

RUIZ-ORTEGA, M. J.; PARRA-REQUENA, G.; GARCÍA-VILLAVERDE, P. M. Do territorial agglomerations still provide competitive advantages? A study of social capital, innovation, and knowledge. International Regional Science Review, v. 39, n. 3, p. 259-290, 2016.

SARACENI, A. V.; ANDRADE JR., P. P.; RESENDE, L. M. Gestão de conhecimento em aglomerados de empresas: rede de valor como instrumento para fortalecer o intercâmbio de conhecimento. Revista Eletrônica de Estratégia \& Negócios, v. 6, n. 1, p. 89-107, 2013.

SILVA, P. M. Aglomerados e turismo: análise da produção científica nacional e internacional sobre o tema. Turismo: Visão e Ação, v. 16, n. 2, p. 338-357, 2014.

SILVEIRA, A.; DREHER, M. T.; ULLRICH, D. R. Desenvolvimento do arranjo produtivo local em turismo sustentável: entendimento dos empreendedores sobre a nova Rússia, Blumenau, SC. Revista de Negócios, v. 10, n. 4, p. 276-287, 2005.

SOUZA, N. J. Desempenho e perspectivas da indústria gaúcha, 1990/2006. In: ENCONTRO DE ECONOMIA - PUCRS, 3., 2006, Porto Alegre. Anais [...]. Porto Alegre: PUCRS, 2006.

TAVARES, B.; ANTONIALLI, M. L.; CALEGARIO, L. L. C.; CASTRO, C. C.; LIMA, C. F. T. A. A. A heterogeneidade entre pequenas empresas: identificando modelos de gestão em uma aglomeração produtiva. Revista de Administração, v. 8, n. 3, p. 515-531, 2015.

TEECE, D. J. Towards an economic theory of the multiproduct firm. Journal of Economic Behavior \& Organization, v. 3, n. 1, p. 39-63, 1982.

THOMAZ, C. J.; BRITO, Z. P. E.; MARCONDES, C. R.; FERREIRA, M. C. F. Benefícios da aglomeração de firmas: evidências do arranjo produtivo de semijoias de Limeira. Revista de Administração, v. 46, n. 2, p. 191-206, 2011.

VENKATRAMAN, N; RAMANUJAM, V. Measurement of business performance in strategy research: A comparison of approaches. Academy of Management Review, v. 11, n. 4, p. 801-814, 1986.

WANG, C. L.; AHMED, P. K. Dynamic capabilities: a review and research agenda. International Journal of Management Reviews, v. 9, n. 1, p. 31-51, 2007.

WERNERFELT; B. A resource-based view of the firm. Strategic Management Journal, v. 5, n. 2, p. 171-180, 1984.

WERNERFELT, B. The use of resources in resource acquisition. Journal of Management, v. 37, n. 5, p. 1.369-1.373, 2011.

WILKE, E. P.; DIETTRICH, L. C. Recursos estratégicos: estudo de caso em hotel UPSCALE. Revista Reuna, v. 25, n. 1, p. 20-34, 2020. 